\title{
Fermi Large Area Telescope observations of high-energy gamma-ray emission from behind-the-limb solar flares
}

\author{
Melissa Pesce-Rollins ${ }^{* a}$, Nicola Omodei ${ }^{b}$, Vahé Petrosian $^{b}$, Wei Liu ${ }^{b}$, Fatima Rubio \\ da Costa $^{b}$ and Alice Allafort ${ }^{b}$ on behalf of the Fermi-LAT Collaboration \\ ${ }^{a}$ Istituto Nazionale di Fisica Nucleare, Sezione di Pisa, I-56127 Pisa, Italy \\ ${ }^{b}$ W.W. Hansen Experimental Physics Laboratory and Physics Department, Stanford University, \\ Stanford, CA 94305, USA \\ E-mail: melissa.pesce.rollinsepi.infn.it \\ nicola.omodeiestanford.edu \\ vahepestanford.edu \\ weiliulmsal.com \\ frubiodstanford.edu \\ allafortestanford.edu
}

Fermi-LAT $>30 \mathrm{MeV}$ observations have increased the number of detected solar flares by almost a factor of 10 with respect to previous space observations. These sample both the impulsive and long duration phases of GOES $\mathrm{M}$ and $\mathrm{X}$ class flares. Of particular interest is the recent detections of three solar flares whose position behind the limb was confirmed by the STEREO-B spacecraft. While gamma-ray emission up to tens of $\mathrm{MeV}$ resulting from proton interactions has been detected before from occulted solar flares, the significance of these particular events lies in the fact that these are the first detections of $>100 \mathrm{MeV}$ gamma-ray emission from footpointocculted flares. We will present the Fermi-LAT, RHESSI and STEREO observations of these flares and discuss the various emission scenarios for these sources and implications for the particle acceleration mechanisms.

The 34th International Cosmic Ray Conference,

30 July- 6 August, 2015

The Hague, The Netherlands

\footnotetext{
* Speaker.
} 


\section{Introduction}

During its first seven years in orbit, the Fermi Large Area Telescope (Atwood et al., 2009) has detected $>30 \mathrm{MeV}$ gamma-ray emission from more than 40 solar flares, nearly 10 times more than EGRET (Thompson et al., 1993) onboard the Compton Gamma-Ray Observatory, GRS (Forrest et al., 1985) onboard the Solar Maximum Mission (SMM) and CORONAS-F (Kuznetsov et al., 2011). The Fermi detections sample both the impulsive (Ackermann et al., 2012a) and the longduration phases (Ackermann et al., 2014) including the longest extended emission ever detected ( 20 hours) from the SOL2012-03-07 GOES X-class flares (Ajello et al., 2014). Fermi-LAT has also provided the first detections of $>100 \mathrm{MeV}$ emission from three occulted solar flares. These observations sample flares from active regions originating from behind both the eastern and western limbs and include an event associated with the second ground level enhancement event (GLE) of the $24^{\text {th }}$ Solar Cycle. These detections present a unique opportunity to diagnose the mechanisms of high-energy emission and particle acceleration in solar flares.

\section{Behind-the-limb flares detected by the Fermi-LAT}

In this section we briefly present the observational overview for these events. Table 1 lists the start times, estimated GOES classes, Active Region (AR) locations, CME speeds and total energy released in $>100 \mathrm{MeV}$ gamma-rays for the three behind-the-limb flares detected by Fermi-LAT.

\begin{tabular}{ccccc}
\hline $\begin{array}{c}\text { Date } \\
(\text { UTC })\end{array}$ & $\begin{array}{c}\text { Estimated GOES } \\
\text { class }\end{array}$ & AR position & $\begin{array}{c}\text { CME speed } \\
\left(\mathrm{km} \mathrm{s}^{-1}\right)\end{array}$ & $\begin{array}{c}\text { Total energy } \\
(\mathrm{ergs})\end{array}$ \\
\hline 2013 Oct 11 07:01 & M4.9 & N21E106 $\left(10^{\circ}\right.$ b.t.l $)$ & 1200 & $3 \times 10^{23}$ \\
2014 Jan 06 07:40 & X3.5 & S8W110 $\left(20^{\circ}\right.$ b.t.l $)$ & 1400 & $5 \times 10^{21}$ \\
2014 Sep 01 11:00 & X2.1 & N14E126 $\left(36^{\circ}\right.$ b.t.l $)$ & 2000 & $2 \times 10^{25}$ \\
\hline \hline
\end{tabular}

Table 1: Behind-the-limb flare properties. The start time of the flare is estimated from STEREO imaging. The GOES class is evaluated using STEREO-observed extreme ultraviolet intensity, as described by (Nitta et al., 2013). The CME speed listed here is the 2nd-order speed at $20 R_{\odot}$ taken from the LASCO online catalog and the total energy released is for the $>100 \mathrm{MeV}$ LAT emission assuming a smooth time profile.

\subsection{SOL2013-10-11}

On 2013 October 11 at 07:01 UT a GOES M1.5 class flare occurred with soft X-ray emission lasting 44 min and peaking at 07:25:00 UT. The left panel of Figure 1 shows the GOES, STEREOB, RHESSI, Fermi Gamma-ray Burst Monitor (GBM; Meegan et al., 2009) and LAT lightcurves of this flare. The LAT detected $>100 \mathrm{MeV}$ emission for $\sim 30$ min with the maximum of the flux occurring between 07:20:00-07:25:00 UT. RHESSI coverage was from 07:08:00-07:16:40 UT, overlapping with Fermi for 9 min. Based on the STEREO-B $195 \AA$ we estimate the GOES class of this flare if the active region had not been occulted to be an M4.9.

Images in the right panel of Figure 1 from the STEREO-B Extreme UltraViolet Imager (EUVI; Wuelser et al., 2004) and the SDO Atmospheric Imaging Assembly (AIA; Lemen et al., 2012) of 
the photosphere indicate that the active region (AR) was $\sim 10^{\circ}$ behind the limb at the time of the flare. From these images we find that the off-limb RHESSI source lies within the $68 \%$ error circle of the LAT emission. This, however, does not exclude that the emission originates from the visible side of the solar disk.
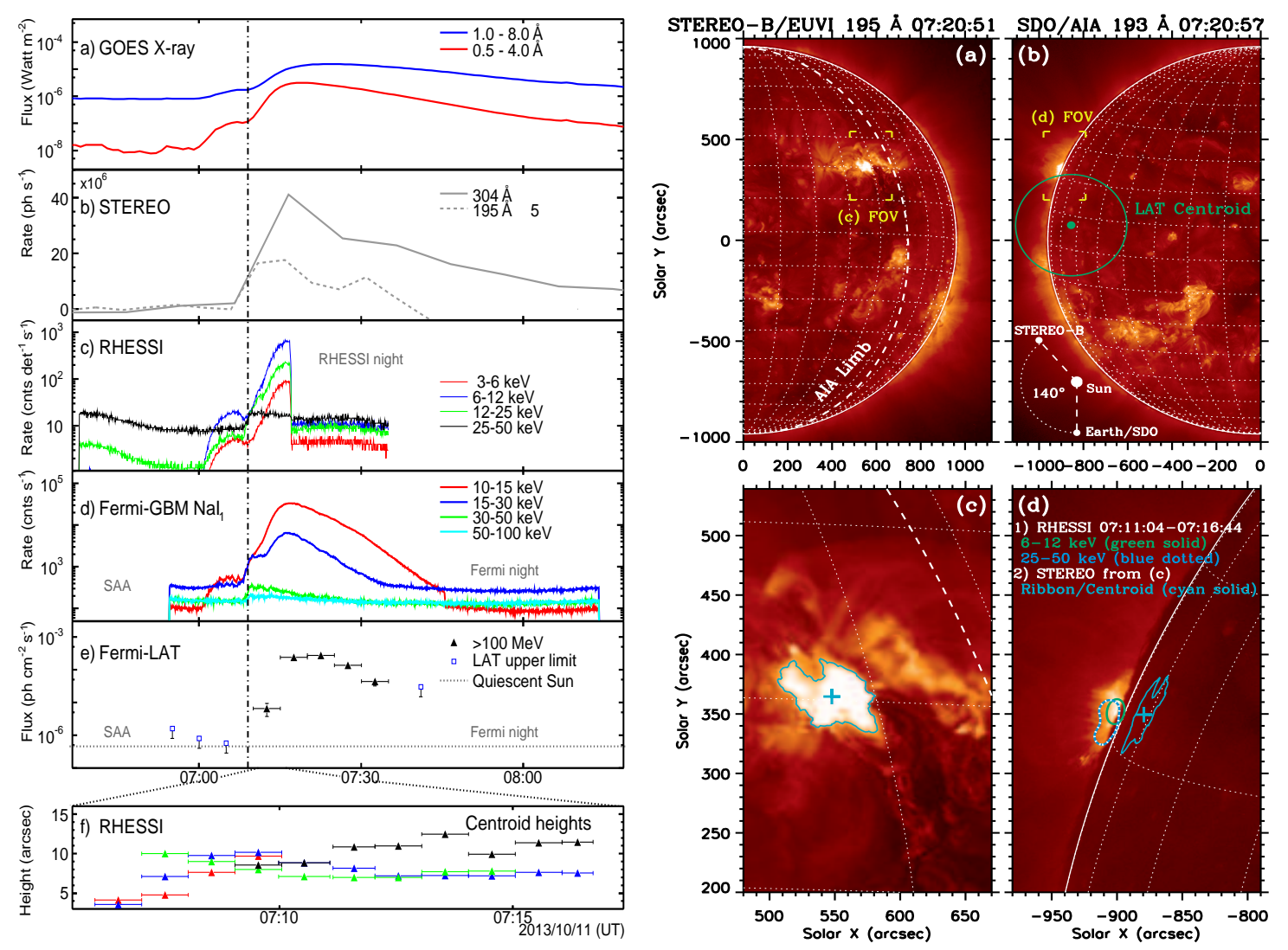

Figure 1: Left panel: Light curves of the 2013 October 11 flare (Pesce-Rollins et al., 2015) as detected by a) GOES, b) STEREO, c) RHESSI, d) GBM, e) LAT, and heights of the RHESSI emission centroid (f) with the same color coding as in c). Fermi exited the South Atlantic Anomaly (SAA) at 06:57:00 UT. The vertical dashed line represents flare start time (7:01 UT). Right panel: STEREO-B (left) and SDO (right) images near the flare peak. The white-dashed line in (a) and (c) represents the solar limb as seen by SDO. The green line in (b) shows the $68 \%$ error circle for the LAT emission centroid. The cyan contour and plus sign in (c) mark the STEREO flare ribbon and its centroid, respectively. Their projected view as seen from the AIA perspective is shown in (d), in which the centroid is located at $10^{\circ}$ behind the limb. The green and blue-dotted contours in (d) show RHESSI sources. The rectangular brackets in (a) and (b) mark the field of view (FOV) for (c) and (d), respectively.

\subsection{SOL2014-01-06}

On 2014 January 6 a solar flare occurred at approximately 07:42:00 UT from the AR located S8W110 ( $\sim 20^{\circ}$ behind-the-limb). Both STEREO spacecraft had a full view of the AR and detected a large filament erupting from the AR starting from 07:44:00 UT. Figure 2 shows the evolution of this filament as seen from the visible solar disk by SDO/AIA 171. LASCO detected a halo CME 
with first C2 appearance at 08:00:00 UT with a 2nd-order Speed at $20 R_{\odot}$ of $1385 \mathrm{~km} / \mathrm{s}$. A ground

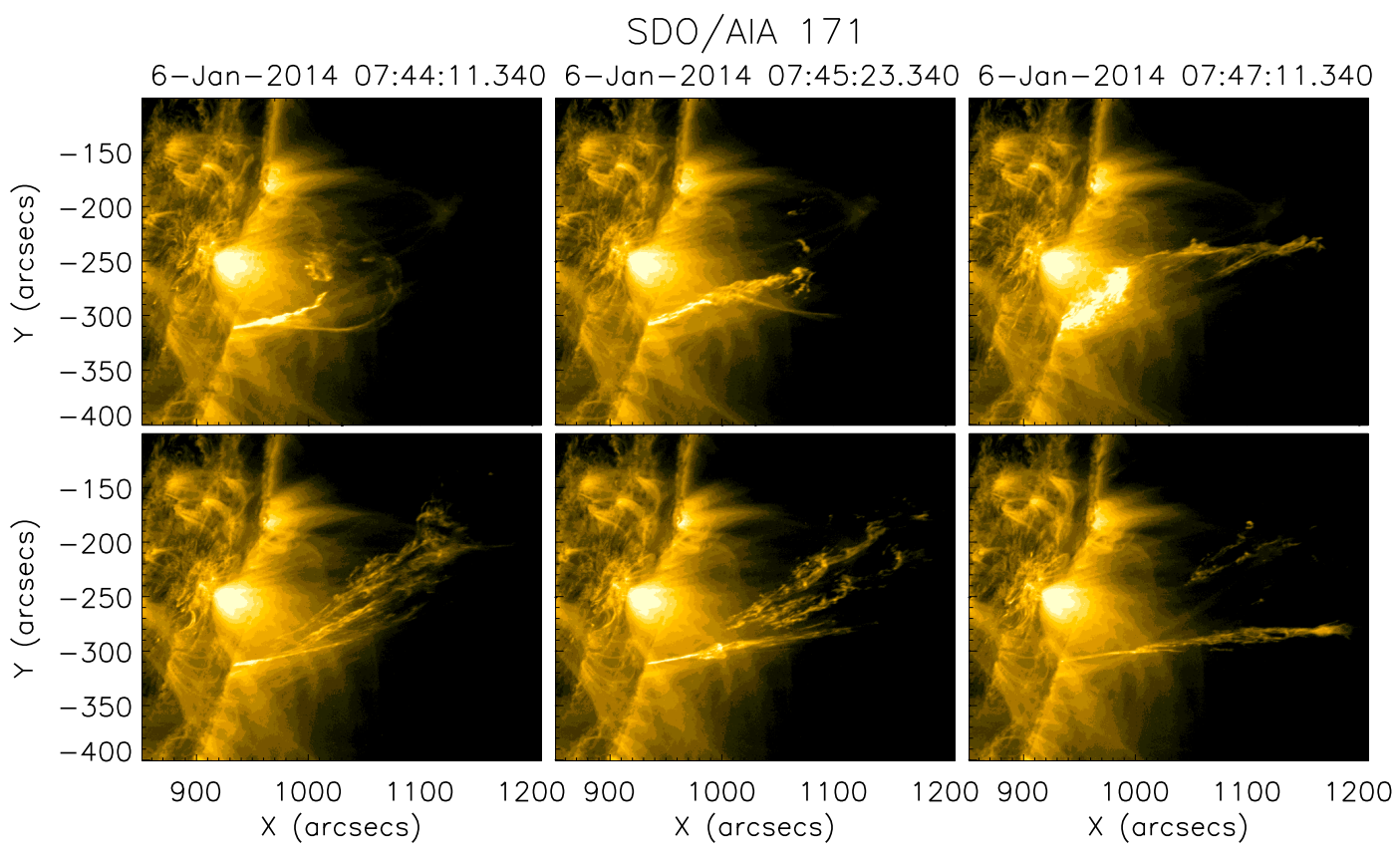

Figure 2: Evolution of the filament eruption associated to SOL2014-01-06 as seen from SDO/AIA 171.

level enhancement (GLE) was also reported in association with this flare making it the second GLE of Solar Cycle 24. Thakur et al. (2014) report that this event (GLE72) was primarily observed by the South Pole neutron monitors with an increase of $\sim 2.5 \%$ over the background level. The increase in the GOES proton flux was evident up to energies $>700 \mathrm{MeV}$. Based on the onset times of the SEPs detected at Earth combined with the onset time of the GLE it is possible to estimate the particle acceleration time at the Sun. We find that a straight line fit to the onset times as a function $1 / \beta$ gives the acceleration time to be on 2014 January 6 at 07:49 \pm 3 minutes UT. This is in agreement with the solar particle release time of 07:47 UT reported by Thakur et al. (2014).

STEREO-B HET data show no significant increase in the proton fluxes during this event indicating that the magnetic connection between the CME and Earth was privileged with respect to that of the STEREO spacecraft. The peak rate detected by STEREO was $2.5 \times 10^{5}$ photons s $^{-1}$ in its $195 \AA$ channel, corresponding to a GOES X3.5 if it had not been occulted.

Upon exiting the the SAA at 07:55:00 UT both detectors onboard Fermi detected emission from this flare. The LAT detected $>100 \mathrm{MeV}$ emission for approximately 20 minutes and the GBM dected emission in the 10's of keV range. No evidence of extended $>100 \mathrm{MeV}$ emission was detected by the LAT. RHESSI detected off-limb emission starting around 8:20 UT (upon exiting the SAA) also in the 10's of keV for over 40 minutes from this flare.

\subsection{SOL2014-09-01}

On 2014 September 1 a bright solar flare occurred from AR 12158 which was located at N14E126 ( $\sim 36^{\circ}$ behind the limb). LASCO detected a halo CME with first C2 appearance at 
11:12:00 UT with a 2nd-order Speed at $20 R_{\odot}$ of $1216 \mathrm{~km} / \mathrm{s}$. A Type II radio burst with an estimated velocity of $2079 \mathrm{~km} / \mathrm{s}$ was also measured in association with this flare. STEREO-B had an unblocked view of the entire flare and detected a maximum rate of $1.7 \times 10^{7}$ photons s $^{-1}$ in its 195 A channel, corresponding to a GOES X2.1 class if it had not been occulted (Nitta et al., 2013). The composite lightcurve of STEREO-B, GOES X-ray, RHESSI, Fermi-GBM and Fermi-LAT data
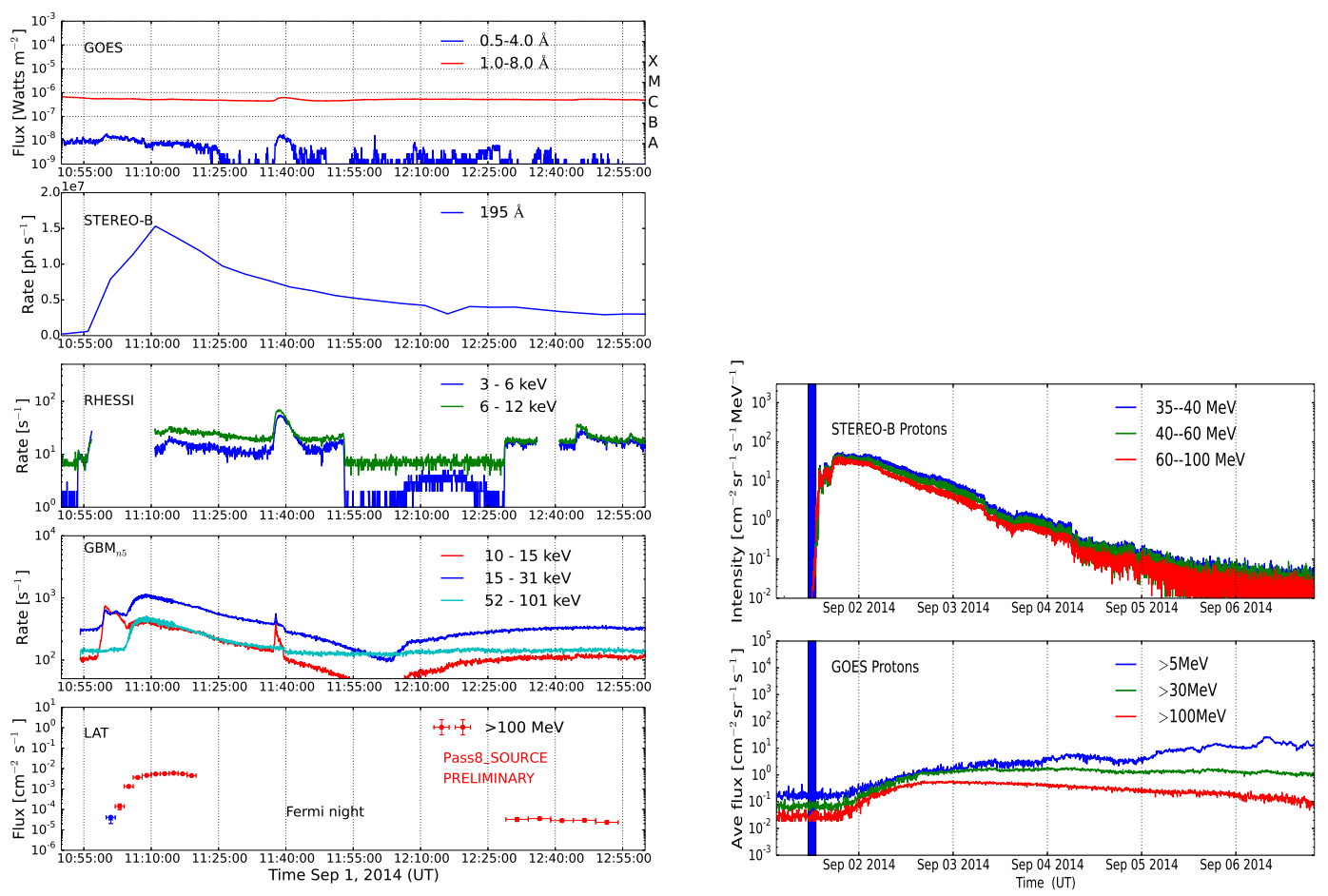

Figure 3: Left panel: Composite lightcurve of STEREO-B EUV, GOES X-ray, RHESSI, Fermi-GBM and Fermi-LAT data for SOL2014-09-01. Right panel: STEREO-B HET proton and GOES SEP proton flux. The blue band in the right hand panel represents the time interval in which the Fermi-LAT detected gamma-ray emission.

for this flare is shown in Figure 3. The high-energy flare emission lasts for $\sim 2$ hours but the LAT detected $>100 \mathrm{MeV}$ emission for $\sim 1$ hour due to the Fermi night between 11:30 and 12:25 UT. The peak LAT flux occurred between 11:10:00-11:15:00 UT. Thanks to improvements provided by the new LAT event selection ( ass $^{1}{ }^{1}$ ) we gained 10 minutes of detected coverage with respect to PasS7_REP of this flare during the impulsive phase. As shown in the left panel of Figure 3 the STEREO-B 60-100 MeV proton flux starts to increase roughly 3 hours after the start of the flare whereas the GOES $>100 \mathrm{MeV}$ proton flux shows an increase roughly 9 hours later.

The GBM detected emission in temporal coincidence with the LAT emission in both the BGO and NaI instruments. RHESSI was in the SAA from 10:55:00 to 11:11:00, upon exiting the SAA it detected emission up to $12 \mathrm{keV}$. Imaging in this time interval with RHESSI shows a source located

\footnotetext{
${ }^{1} \mathrm{~A}$ summary of the Fermi-LAT Pass 8 performance can be found here http://www.slac.stanford.edu/exp/glast/groups/canda/lat_Performance.htm.
} 
at approximately heliocentric coordinates $\left[-900^{\prime \prime}, 400^{\prime \prime}\right]$ which is in the vicinity of the LAT time integrated $>100 \mathrm{MeV}$ emission centroid. If the RHESSI source is the looptop of the behind-thelimb flare then the minimum height needed for this source to be visible from $\sim 40^{\circ}$ behind-the-limb would be $\sim 10^{10} \mathrm{~cm}$. In fact, X-ray emission from a flare located $\sim 40^{\circ}$ behind-the-limb has been detected before by RHESSI (Krucker et al., 2007).

The LAT measured 18 P8R2_SOURCE photons with energies $>1 \mathrm{GeV}$ and reconstructed direction less than $1^{\circ}$ from the center of the solar disk. 15 of these, including a $3.5 \mathrm{GeV}$ photon, arrived between 11:08 and 11:20 UT.

\section{Discussion}

We present the Fermi-LAT observations of high-energy gamma-ray emission from three behindthe-limb solar flares (SOL2013-10-11, SOL2014-01-06 and SOL2014-09-01) together with multiwavelength data from STEREO, GOES and RHESSI. Based on our work in Pesce-Rollins et al. (2015) we can exclude the scenario in which the gamma-ray emission occurred at the AR position due to the large optical depths involved. Although the scenario in which the emission originates from the Corona is not entirely excluded, we suggest that the gamma-ray emission detected by the LAT was caused by protons accelerated at the CME-driven shock. In this scenario, also proposed by Cliver et al. (1993), the particles are accelerated on open field lines and can either precipitate to the visible disk and produce gamma-ray emission or escape to be observed as SEPs. Further studies on the precipitation rate of the protons producing the observed gamma-ray emission from these behind-the-limb flares is currently underway together with detailed comparison of the flare characteristics with other on-disk Fermi-LAT detections (paper in preparation).

\section{Acknowledgments}

The Fermi LAT Collaboration acknowledges support from a number of agencies and institutes for both development and the operation of the LAT as well as scientific data analysis. These include NASA and DOE in the United States, CEA/Irfu and IN2P3/CNRS in France, ASI and INFN in Italy, MEXT, KEK, and JAXA in Japan, and the K. A. Wallenberg Foundation, the Swedish Research Council and the National Space Board in Sweden. Additional support from INAF in Italy and CNES in France for science analysis during the operations phase is also gratefully acknowledged. V.P, W.L and F.R.d.C are supported by NASA grants NNX14AG03G, NNX13AF79G and NNX12AO70G. M.P.R is supported by NASA grant NNX13AC47G.

\section{References}

Ackermann, M., Ajello, M., Allafort, A., et al. 2012a, Astrophys. J., 745, 144

Ackermann, M., Ajello, M., Albert, A., et al. 2014, The Astrophysical Journal, 787, 15

Ajello, M. A. A., Allafort, A., Baldini, L., et al. 2014, The Astrophysical Journal, 789, 20

Atwood, W. B.Abdo, A. A., Ackermann, M., Ajello, M., et al. 2009, Astrophys. J., 697, 1071 
Barat, C., Trottet, G., Vilmer, N., et al. 1994, Astrophys. J. Lett., 425, L109

Chertok, I. M., Belov, A. V., \& Grechnev, V. V. 2015, ArXiv e-prints

Cliver, E. W, Kahler, S. W. \& Vestrand, W. T. 1993, International Cosmic Ray Conference, 3, 91

Forrest, D. J., Vestrand, W. T., Chupp, E. L., et al. 1985, International Cosmic Ray Conference, 4, 146

Krucker, S., White, S. M., \& Lin, R. P. 2007, Astrophys. J. Lett., 669, L49

Kuznetsov, S., Kurt, V., Yushkov, B., Kudela, K., \& Galkin, V. 2011, Solar Physics, 268, 175

Lemen, J., Title, A., Akin, D., et al. 2012, Solar Physics, 275, 17

Meegan, C., Lichti, G., Bhat, P. N., et al. 2009, Astrophys. J., 702, 791

Nitta, N. V., Aschwanden, M. J., Boerner, P. F., et al. 2013, Sol. Phys., 288, 241

Pesce-Rollins, M., Omodei, N., Petrosian, V., et al. 2015, Astrophys. J. Lett., 805, L15

Thakur, N., Gopalswamy, N., Xie, H., et al. 2014, Astrophys. J. Lett., 790, L13

Thompson, D. J., Bertsch, D. L., Fichtel, C. E., et al. 1993, Astrophys. J. Supp. Series, 86, 629

Vestrand, W. T., \& Forrest, D. J. 1993, Astrophys. J. Lett., 409, L69

Vilmer, N., Trottet, G., Barat, C., et al. 1999, Astronomy and Astrophysics, 342, 575

Wuelser, J., Lemen, J. R., Tarbell, T. D., et al. 2004, Society of Photo-Optical Instrumentation Engineers (SPIE) Conference Series 5171 (2004) 